\title{
Specialization of trophic position and habitat use by sticklebacks in an adaptive radiation
}

\author{
Blake Matthews, ${ }^{1,5}$ Kerry B. Marchinko, ${ }^{2}$ Daniel I. Bolnick, ${ }^{3}$ and Asit Mazumder ${ }^{4}$ \\ ${ }^{1}$ EAWAG, Aquatic Ecology Department, Center for Ecology, Evolution and Biogeochemistry, Seestrasse 79, \\ Kastanienbaum CH-6047, Switzerland \\ ${ }^{2}$ Fred Hutchinson Cancer Research Center, 1100 Fairview Avenue N, Seattle, Washington 98109-1024 USA \\ ${ }^{3}$ Howard Hughes Medical Institute and Section of Integrative Biology, University of Texas, \\ One University Station C0930, Austin, Texas 78712 USA \\ ${ }^{4}$ Water and Aquatic Sciences Research Program, University of Victoria, Department of Biology, P.O. Box 3020, Station CSC, \\ Victoria, British Columbia V8W3N5 Canada
}

\begin{abstract}
Divergence in habitat use among closely related species is a common characteristic of adaptive radiations. Large differences in the size structure of prey between habitats could strengthen disruptive selection on generalist predators and lead to a divergence in trophic position among species in an adaptive radiation. Using threespine stickleback (Gasterosteus aculeatus) in freshwater lakes as a model system, we examined whether divergence in habitat use coincides with shifts in trophic position. We examined the habitat use and trophic position of individual sticklebacks from divergent lake environments that have only one stickleback species (allopatric lakes) and from lakes that have a pair of benthic and limnetic stickleback species (sympatric lakes). In two sympatric lakes, the limnetic species had a higher trophic position than the benthic species, and in both allopatric and sympatric lakes, sticklebacks specializing on pelagic prey had a higher trophic position for a given size than sticklebacks specializing on benthic prey. Furthermore, the trophic position of pelagic specialists was correlated with individual variation in their gill raker length. Our results indicate that gill raker length is an important trait that underlies differentiation in both habitat use and trophic position among stickleback species, populations, and individuals.
\end{abstract}

Key words: adaptive radiation; divergent selection; eco-evolutionary dynamics; ecological speciation; functional morphology; habitat specialization; individual specialization; lake food webs; pelagic foraging; stickleback; trophic position.

\section{INTRODUCTION}

The ecological factors that underlie phenotypic differentiation and determine the strength of divergent and disruptive selection remain poorly understood (Abrams et al. 2008). Divergent selection results from both biotic and abiotic differences among foraging environments (Losos et al. 1998, Landry et al. 2007) and contributes to variation in the foraging traits and habitat preferences of consumers (Schluter and Nagel 1995, Schluter 2000, Knudsen et al. 2007). Ample theoretical and experimental evidence suggests that both competition and predation affect the strength of divergent selection (Schluter 2000, Rundle et al. 2003, Bolnick 2004); however, the context of species' interactions (e.g., food web configuration) is also important because not all environments are equally conducive to generating disruptive selection (Bolnick and Lau 2008). For example, disruptive selection on the foraging traits of sticklebacks is strongest in intermediate sized lakes that

Manuscript received 16 February 2009; revised 3 June 2009; accepted 10 June 2009; final version received 27 July 2009. Corresponding Editor: D. E. Schindler.

${ }^{5}$ E-mail: blake.matthews@eawag.ch have a similar proportion of littoral and pelagic habitat (Bolnick and Lau 2008). Similarly, phenotypic differences among whitefish morphs are largest in lakes with small zooplankton, suggesting that disruptive selection may be stronger when adjacent habitats have prey communities with contrasting size structures (Landry et al. 2007).

Integrating food web theory with the study of adaptive radiation can reveal new insights about how food web structure affects species diversification, and vice versa (Schluter 2000, Harmon et al. 2009). Food web modeling has a long history in ecology (Dunne 2005) but theoreticians have only recently begun to explore the evolutionary dimensions of food web structure (Loeuille and Loreau 2005, Martinez 2005, Jones et al. 2009). In general terms, adaptive radiations can lead to an increase in both the horizontal diversity and vertical diversity of food webs (Duffy et al. 2007). At low trophic positions, consumers compete for resources from a single trophic level (e.g., plants), and this interspecific competition can drive phenotypic differentiation among consumer species, such as in finches (Grant 1986), leading to an increase in the number of linkages between adjacent trophic levels (i.e., an increase in horizontal diversity). At higher trophic 
positions in the food chain, predators compete for prey species that have a broad range of trophic positions. As a result, antagonistic interactions other than competition, such as apparent competition, shared predation, or intraguild predation could drive phenotypic differentiation among consumer species (Schluter 2000). In adaptive radiations of predators, such as in cichlid radiations (Fryer and Iles 1972), the evolution of foraging traits, such as body size, mobility, or gape width, may affect the vertical diversity of food webs by shaping the patterns of trophic linkages between predators and prey.

The structural variation of food chains among foraging habitats is often overlooked as a source of disruptive selection (Brose 2006, Rooney et al. 2008). The landscape theory of food web structure posits that food webs consist of multiple food chains, or energy channels, that are supported by distinct sources of primary production (Polis and Strong 1996) and are linked by mobile consumers that adaptively forage among them (Rooney et al. 2006). Predators at high trophic positions tend to forage in multiples habitats and exploit prey from distinct food chains, effectively linking food chains together into a food web (Polis and Strong 1996). Variation in the traits and habitats of primary producers causes food chains to receive asynchronous pulses of energy at their base, leading to food chains with organisms (and prey) that differ widely in their size structure, trophic position, and rates of biomass turnover (McCann et al. 2005, Rooney et al. 2008). As a result, generalist predators that feed in multiple habitats can face strong foraging tradeoffs that may strengthen disruptive selection (Robinson 2000, Abrams et al. 2008). Over evolutionary time, the asymmetries that confer long term stability to food webs (Rooney et al. 2006) may eventually cause divergence in the foraging traits of predators (Landry et al. 2007).

Asymmetries in the trophic size structure of prey in different lake habitats may have contributed to the differentiation of multiple fish species into benthic and pelagic forms in numerous northern temperate lakes (Robinson and Wilson 1994). Because predator-prey relationships tend to be more size-structured in pelagic compared to benthic food chains (Diehl 1992, Blumenshine et al. 2000), relationships between body size and trophic position may be steeper in communities of pelagic compared to benthic prey. If this structural variation among food chains is important for the diversification of fish, we would expect a comparative study to produce the following three patterns. First, pelagic fish should exhibit stronger relationships between their body size and trophic position because they specialize on prey from a more size-structured food chain than benthic fish. In addition, we would expect pelagic fish to have a higher trophic position than benthic fish because prey of similar sizes have a higher trophic position in pelagic environments (Rooney et al.
2006). Second, a heritable foraging trait, such as gill raker length, should underlie the differentiation in trophic position between pelagic and benthic forms, and serve as an ecological basis for the divergence (Schluter 2000). Differentiation in trophic position could be observed among closely related species, among populations, or among individuals within a population. For example, in fish populations where some members specialize on the pelagic food chain and others on the benthic chain, individual variation in gill raker length might correlate with trophic position. Trophic position can thus be considered a phenotypic trait of individuals, in the sense that it is a measurable quantity that varies among individuals as an emergent consequence of morphological variation playing out in a given ecological community. To the extent that the underlying morphology is heritable, trophic position can evolve in response to selection. Finally, there should be evidence for disruptive selection in fish populations with respect to both trophic position and habitat use. In the current study, we address the first two predicted patterns.

Studies of adaptive radiations rarely quantify shifts in trophic position (Schluter 2000), but such shifts might be common in systems, such as lakes, where consumers forage on multiple food chains that differ in their size structure and trophic interactions (Robinson and Wilson 1994, Zanden and Vadeboncoeur 2002, Rooney et al. 2008). Threespine stickleback (Gasterosteus aculeatus) are good model organisms to examine whether habitat specialization is associated with shifts in trophic position during an adaptive radiation. Benthic and limnetic species of stickleback have diverged in multiple traits that are associated with specialization to different foraging habitats (Hatfield and Schluter 1999, Vamosi and Schluter 2004). Limnetics have longer and more numerous gill rakers and feed more efficiently on small pelagic zooplankton than benthics (Schluter 1993). This pattern of adaptation to pelagic and littoral foraging habitats also appears in allopatric populations from divergent lake environments (Lavin and McPhail 1985). For example, sticklebacks tend to have more and longer gill rakers in large, deep lakes with extensive pelagic foraging habitat (Lavin and McPhail 1985). Within stickleback populations, gill raker length is often under disruptive selection (Bolnick 2004, Bolnick and Lau 2008) and partially underlies diet variation among individuals (Svanback and Bolnick 2007, Bolnick et al. 2008). Given that gill raker length is associated with habitat specialization by stickleback, does it also underlie their specialization with respect to trophic position? The trophic position of an individual results from an interaction between the community structure of the environment in which it forages, and its phenotypic ability and inclination to select particular subsets of the available prey. Consequently, among-population variation in trophic position may be driven by differences in either prey availability, the stickleback's phenotype, or feedback loops between these (Harmon et al. 2009). On 

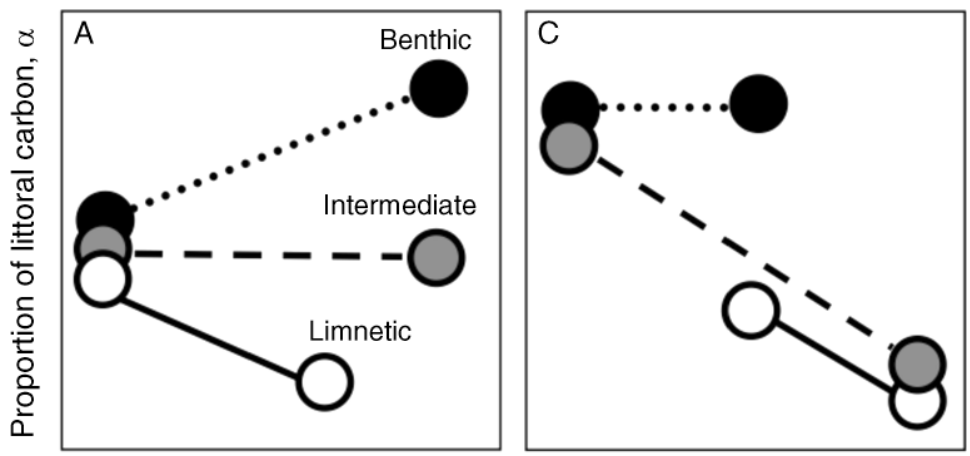

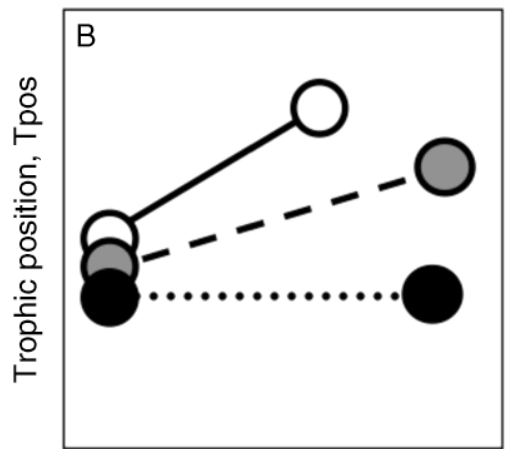

Body size

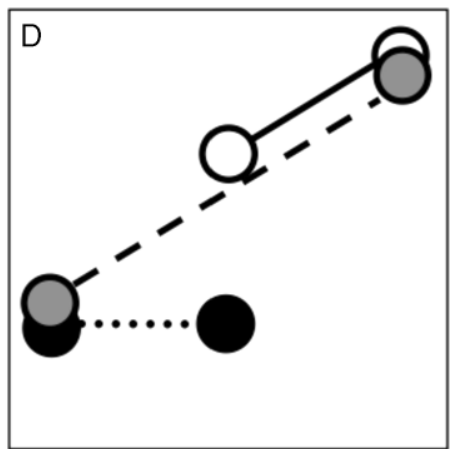

Pelagic foraging trait

FIG. 1. Predictions for the relationship between trophic position (Tpos), the proportion of littoral carbon in the diet of sticklebacks $(\alpha)$, and variation in body size and feeding morphology.

the other hand, within-population variation in trophic position (or differences between co-occurring species) must reflect phenotypic variation because the prey community is shared.

Stable isotope analysis (SIA) is a useful tool for measuring the resource specialization of sticklebacks. In lakes, the $\delta^{13} \mathrm{C}$ of stickleback prey differs strongly among littoral and pelagic habitats, such that we can quantify individual-based variation in habitat use and associate this with morphological variation. Furthermore, $\delta^{15} \mathrm{~N}$ allows us to discern whether individual sticklebacks specialize on prey from different trophic positions (Matthews and Mazumder 2005). SIA is better suited for these applications than traditional gut content analysis because isotopes record a longer period of dietary history and provide temporal consistency to measures of individual specialization (Bolnick et al. 2003, Matthews and Mazumder 2004, Araujo et al. 2007). Furthermore, analyzing only the gut contents of fish will typically underestimate the amount of differentiation in trophic position among zooplankton prey and, hence, among individual fish (Matthews and Mazumder 2005). Here, we use stable isotopes of carbon $\left(\delta^{13} \mathrm{C}\right)$ to examine individual variation in habitat use by sticklebacks, and stable isotopes of nitrogen $\left(\delta^{15} \mathrm{~N}\right)$ to measure stickleback trophic position. By linking isotopic variation with morphometrics we investigate which foraging traits underlie diet variation among individuals (Matthews and Mazumder 2004, Araujo et al. 2007).
Using both stable isotope analysis and morphometrics we tested the following two predictions. First, we tested for size-based shifts in habitat and trophic-position specialization by examining relationships between stickleback body size, trophic position (Tpos), and the proportion of dietary carbon from littoral sources $(\alpha$; Fig. 1A, B). We predicted that limnetics and benthics would exhibit size-based specialization on pelagic and benthic habitats, respectively. In allopatric populations, we expected larger stickleback to specialize on resources from the most common habitat, as determined by lake morphometry. That said, we anticipated that relationships between stickleback body size and $\alpha$ might not be apparent in allopatric stickleback populations, either because the population consists of habitat generalists or an even mixture of both benthic and pelagic specialists. In addition, we tested whether shifts in habitat use were accompanied by parallel or divergent shifts in trophic position. Because we expected there to be steeper relationships between prey size and trophic position in pelagic habitats (Diehl 1992), we predicted that sticklebacks specializing on pelagic habitats would also exhibit a steeper relationship between their body size and trophic position (Fig. 1B).

Second, we tested for individual specialization in both habitat use and trophic position (Tpos) by examining the relationship between fish morphology, $\alpha$, and Tpos (Bolnick et al. 2003). Because the foraging traits that underlie individual specialization may differ among 
environments (Robinson 2000), we expected that relationships between gill raker length (GRL) and Tpos would differ among habitats, as would relationships between GRL and $\alpha$. For sticklebacks that forage in pelagic environments, we expected that individuals with relatively long gill rakers would rely less on littoral carbon sources (Fig. 1C) and have a higher trophic position (Fig. 1D). In contrast, we expected that stickleback foraging almost exclusively in benthic environments would show no relationship between their gill raker length and either $\alpha$ or Tpos (Fig. 1C, D).

\section{Methods}

Study sites.-We collected threespine sticklebacks from six lake populations on islands off the west coast of British Columbia, Canada. Our study lakes (Appendix), include one population with a "limnetic-like" phenotype (Kennedy, KEN), one population with an intermediate phenotype (Cranby, CRA), two lakes with a pair of benthic and limnetic species (Paxton, PAX, and Priest, PRI), and one pair of lakes whose populations inhabit divergent lake habitats ("limnetic-like" in Ormond Lake, ORM, and "benthic-like" in Dugout, DUG) but exhibit little phenotypic divergence, presumably because of high gene flow (Bolnick et al. 2008). Despite some migration between Ormond and Dugout Lakes, individuals caught in Ormond have slightly longer and more numerous gill rakers than those caught in Dugout (Bolnick et al. 2008), but the difference in mean gill raker lengths (GRL) between ORM and DUG populations is only $20 \%$ of the average divergence in GRL between limnetics and benthics in PAX and PRI.

Stable isotope analysis.-We used minnow traps and dip nets to collect sticklebacks from the littoral habitat of each lake during the breeding season (May-July). Both benthic and limnetic stickleback nest in the littoral habitat. The lakes on Texada Island (Cranby, Paxton, and Priest) were sampled in June 2004, Kennedy Lake was sampled in May 2004, and Ormond and Dugout lakes were sampled in June 2005. We also concurrently sampled mussels and other littoral invertebrates using a dip net, Eckman grabber, and snorkel. All tissue samples were immediately frozen and stored at $-80^{\circ} \mathrm{C}$. For isotope analysis, we used the foot tissue from mussels, all of the soft tissue from snails, and the dorsal white muscle from sticklebacks. All tissue was freeze dried and ground to a fine powder with either a mortar and pestle or a ball mill (Spex Industries 8000; Edison, New Jersey, USA). Isotope samples were run on a Finnigan Delta Plus Advantage stable isotope ratio mass spectrometer at the University of Victoria.

We used the following equations to model the trophic position of sticklebacks, following Post (2002):

$$
\begin{aligned}
\text { Tpos }=\lambda_{\text {base }}+ & \left(\delta^{15} \mathrm{~N}_{\text {stickle }}-\left[\delta^{15} \mathrm{~N}_{\text {lit }} \times \alpha+\delta^{15} \mathrm{~N}_{\text {pel }}\right.\right. \\
& \times(1-\alpha)]) / \Delta_{\mathrm{N}}
\end{aligned}
$$

$$
\alpha=\left(\delta^{13} \mathrm{C}_{\text {stickle }}-\delta^{13} \mathrm{C}_{\mathrm{pel}}\right) /\left(\delta^{13} \mathrm{C}_{\text {lit }}-\delta^{13} \mathrm{C}_{\mathrm{pel}}\right)
$$

where $\lambda_{\text {base }}$ is the trophic position $\left(\lambda_{\text {base }}=2\right)$ of the consumers that represent the pelagic baseline (pel; e.g., mussels) and littoral baseline (e.g., snails) in each lake, and $\Delta_{\mathrm{N}}$ is the average trophic enrichment obtained from previous studies $\left(\Delta_{\mathrm{N}}=3.4\right.$; as in Post 2002), and $\alpha$ represents the proportion of carbon from littoral sources in the diet of sticklebacks. We could not find any snails in Kennedy Lake, so we collected juvenile Peamouth Chub (39-55 mm) from the littoral habitat to represent the $\delta^{13} \mathrm{C}_{\text {lit }}$, and used mussels for $\delta^{15} \mathrm{~N}_{\mathrm{lit}}$ and $\delta^{15} \mathrm{~N}_{\text {pel. }}$. To ensure this did not bias our analyses, we did a survey of snails and mussels from 11 lakes on Vancouver Island nearby our study sites. As found in a previous study (Post 2002), our survey confirmed that the $\delta^{15} \mathrm{~N}$ of mussels was not significantly different from that of snails $\left(\delta^{15} \mathrm{~N}_{\text {mus-sna }}=-0.23, \mathrm{SD}=0.78\right.$; paired $t$ test, $t=$ $-0.92, P=0.38)$. Overall, the differences in trophic position among sticklebacks are qualitatively similar if we only use mussels to model trophic position (B. Matthews, unpublished data). We did not use any lipid normalization techniques in our study (Kiljunen et al. 2006) because variation in lipid content among the stickleback populations was negligible (stickleback C:N $=3.96, \mathrm{SD}=0.2$ ).

Morphological measurements of sticklebacks.-In each population, we measured standard length, head length, body depth, lower jaw length, snout length, eye width, gill raker number, and length of the three longest gill rakers (Table 1). Principal components analysis (PCA) was performed on the correlation matrix of the logtransformed morphometric data. To investigate morphological variation among lakes, we included all individuals in the analysis and retained the factor scores for the first and second principal components. The first PC explained variation in the length of gill rakers and the second PC explained variation in body size (Table 2). The PCA helped account for allometric differences among lake populations and allowed us to test the predictions illustrated in Fig. 1. Specifically, we did an analysis of covariance (ANCOVA) to determine how the slope of the relationship between stickleback morphology (PC axes) and isotopic variation (e.g., Tpos and $\alpha$ ) differed among populations (Table 3). The PCA allowed us to evaluate, for example, whether trophic position was correlated with a measure of gill raker length (PC1) that was orthogonal to variation in stickleback body size (PC2). We used untransformed data in our ANCOVA analysis and assessed normality using Q-Q plots. All analyses were done in R (v. 2.7.1; R Development Core Team 2008).

\section{RESULTS}

\section{Morphological and ecological niche variation among lakes}

The stickleback populations and species in our study reflect a wide range of phenotypes found in diverse lake environments (Lavin and McPhail 1985). These popu- 
TABLE 1. Mean (with SD in parentheses) for morphology and isotopes for each stickleback population.

\begin{tabular}{|c|c|c|c|c|c|c|c|c|}
\hline Parameter & CRA & PAX-B & PAX-L & PRI-B & PRI-L & ORM & DUG & KEN \\
\hline Number of fish & 86 & 47 & 44 & 46 & 44 & 30 & 30 & 75 \\
\hline$\delta^{13} \mathrm{C}(\%)$ & $-30.6(1.9)$ & $-30.1(1.3)$ & $-31.8(0.9)$ & $-26.4(1.6)$ & $-30.7(0.9)$ & $-25.7(2.1)$ & $-24.6(1.4)$ & $-27.2(1.5)$ \\
\hline$\alpha \dagger$ & $0.5(0.2)$ & $0.7(0.2)$ & $0.4(0.2)$ & $0.6(0.2)$ & $0.1(0.1)$ & $0.6(0.3)$ & $1.0(0.1)$ & $0.1(0.1)$ \\
\hline$\delta^{15} \mathrm{~N}(\%)$ & $11.3(0.6)$ & $11.7(0.9)$ & $12.7(1.3)$ & $10.4(0.4)$ & $12.1(0.5)$ & $8.2(0.7)$ & $7.2(0.4)$ & $9.0(0.4)$ \\
\hline Tpost & $3.6(0.2)$ & $3.6(0.2)$ & $3.8(0.4)$ & $3.5(0.1)$ & $3.7(0.1)$ & $3.8(0.2)$ & $3.5(0.1)$ & $3.7(0.2)$ \\
\hline Standard length $(\mathrm{mm})$ & $47.9(6.9)$ & $51.9(5.7)$ & $41.9(4.3)$ & $49.2(8.4)$ & $45.6(2.5)$ & $45.7(8.4)$ & $48.3(6.2)$ & $51.9(4.2)$ \\
\hline Head length (mm) & $15.5(2.4)$ & $17.6(2.1)$ & $14.0(2.0)$ & $15.8(3.0)$ & $14.5(1.3)$ & $14.4(2.7)$ & $14.9(2.0)$ & $16.6(1.6)$ \\
\hline Body depth (mm) & $10.3(1.6)$ & $11.6(1.5)$ & $8.1(0.7)$ & $10.8(2.3)$ & $8.6(0.7)$ & $10.3(2.0)$ & $10.8(1.3)$ & $11.4(1.1)$ \\
\hline Jaw length (mm) & $3.4(0.7)$ & $3.6(0.5)$ & $2.6(0.5)$ & $3.3(0.9)$ & $3.1(0.5)$ & $4.0(0.8)$ & $4.2(0.6)$ & $4.6(0.5)$ \\
\hline Snout length $(\mathrm{mm})$ & $4.4(0.9)$ & $5.1(0.7)$ & $4.2(0.8)$ & $4.6(1.1)$ & $4.7(0.7)$ & $4.0(1.0)$ & $4.2(0.6)$ & $4.8(0.7)$ \\
\hline Eye width $(\mathrm{mm})$ & $4.1(0.7)$ & $4.5(0.5)$ & $3.9(0.5)$ & $4.2(0.8)$ & $4.2(0.4)$ & $4.1(0.7)$ & $4.1(0.5)$ & $4.6(0.4)$ \\
\hline Raker number & $18.2(1.8)$ & $16.0(1.9)$ & $22.6(1.9)$ & $16.5(2.0)$ & $21.8(2.1)$ & $21.6(1.4)$ & $21.2(1.5)$ & $22.9(1.3)$ \\
\hline Mean raker length $(\mathrm{mm})$ & $1.0(0.2)$ & $0.8(0.1)$ & $1.2(0.1)$ & $0.5(0.1)$ & $1.1(0.1)$ & $0.7(0.2)$ & $0.6(0.1)$ & $1.4(0.2)$ \\
\hline
\end{tabular}

Note: The populations are Cranby Lake (CRA), Paxton Lake benthic (PAX-B) and limnetic (PAX-L), Priest Lake benthic (PRIB) and limnetic (PRI-L), Ormond Lake (ORM), Dugout Lake (DUG), and Kennedy Lake (KEN).

$\dagger$ The proportion of dietary carbon from littoral sources.

† Trophic position.

lations vary widely in two traits that are strongly associated with foraging efficiency in pelagic habitats, namely their average gill raker number and length (Table 1).

On average, sticklebacks derived $45 \%(\mathrm{SD}=31, N=$ 403 fish) of their carbon from littoral sources, but $\alpha$ differed significantly among populations (Table $1 ; F_{7,395}$ $=106.3, P<0.001)$. The average trophic position of sticklebacks was $3.6(\mathrm{SD}=0.23, N=403$ fish), but this also varied significantly among populations (Table 1; $\left.F_{7,395}=13.2, P<0.001\right)$. In sympatric populations, limnetics had a higher trophic position than benthics (Paxton, Tpos ${ }_{\text {lim-ben }}=0.19, t=2.9, P<0.001$; Priest. Tpos $\left._{\text {lim-ben }}=0.20, t=7.2, P<0.001\right)$, and relied on about $25-60 \%$ less littoral carbon than did benthics (Paxton, $\alpha_{\text {lim-ben }}=-0.27, t=7.4, P<0.001$; Priest, $\left.\alpha_{\text {lim-ben }}=-0.54, t=-16.4, P<0.001\right)$.

The pattern of ecological differentiation between limnetics and benthics was similar to that observed among other stickleback populations from divergent lake environments. Sticklebacks from Kennedy Lake had a high trophic position, consistent with their limnetic-like phenotype (Fig. 2), while sticklebacks from Cranby Lake had an intermediate trophic position and $\alpha$, matching their intermediate morphology. Despite very similar morphologies between sticklebacks in Ormond and Dugout (which experience high reciprocal migration rates), Ormond sticklebacks had a higher trophic position and relied much less on littoral carbon sources than Dugout sticklebacks $\left(\right.$ Tpos $_{\text {Orm-Dug }}=0.31$, $t=7.7, P<0.001 ; \alpha_{\text {Orm-Dug }}=-0.35, t=-6.7, P<$ $0.001)$. Indeed, the difference in trophic position between Ormond and Dugout was at least as great as that observed between sympatric species pairs, despite the much weaker morphological divergence.

\section{Relationships between size, morphology, and isotopes}

The proportion of littoral carbon $(\alpha)$ in stickleback diets increased with body size in both benthic species (Paxton and Priest benthics) and in Ormond Lake
(Table 3), but tended to decline with body size in both limnetic species (Paxton and Priest limnetics) and in stickleback from Kennedy and Cranby Lakes (Fig. 3A; Table 3). Intriguingly, this size-based divergence in habitat use (Fig. 3A) was also associated with a sizebased divergence in the trophic position of stickleback (Fig. 3B). The trophic position of stickleback increased with body size in limnetics (PAX, PRI) and in populations with "limnetic-like" phenotypes (ORM, KEN) but not in populations with either benthics (PAX, PRI), a "benthic-like" phenotype (DUG), or an intermediate (CRA) phenotype (Fig. 3; Table 3).

Correlations between isotopes and morphology revealed that individual stickleback within a given population specialized in both their habitat use and trophic position (Fig. 3C, D). In general, individuals with longer gill rakers relied less on littoral carbon sources (i.e., a negative relationship between PC1 and $\alpha$; Fig. 3C), though the reverse was true for benthic populations (Table 3). Furthermore, in all populations, individuals with relatively long gill rakers had higher trophic positions (Table 3), but this relationship was weakest for benthics from Paxton and Priest Lake $(P=$ 0.12 and $P=0.07$, respectively; Table 3$)$. The strong association between trophic position and gill raker

TABle 2. Principal components analysis for all populations.

\begin{tabular}{lcc}
\hline \hline \multicolumn{1}{c}{ Trait } & PC1 & PC2 \\
\hline Standard length & 0.055 & 0.314 \\
Head length & 0.068 & 0.352 \\
Body depth & 0.027 & 0.41 \\
Lower jaw length & 0.125 & 0.56 \\
Snout length & 0.104 & 0.41 \\
Eye width & 0.07 & 0.29 \\
Gill raker number & 0.128 & -0.11 \\
Gill raker length (1) $\dagger$ & 0.513 & -0.09 \\
Gill raker length (2) $\dagger$ & 0.568 & -0.09 \\
Gill raker length (3) $\dagger$ & 0.599 & -0.12 \\
Percentage variance & 40.1 & 24.0 \\
\hline
\end{tabular}

$\dagger$ These are the lengths of the longest (1), second longest (2), and third longest (3) gill rakers. 
TABLE 3. Each column is a model summary, with slope coefficients (for populations) and $P$ values (for $F$ values) in parentheses, for the relationship between trophic position (Tpos) or proportion of dietary carbon from littoral sources $(\alpha)$ and the first two principal components of variation in morphology, namely, gill raker length (GRL) and size.

\begin{tabular}{|c|c|c|c|c|}
\hline Parameter & $\begin{array}{c}\alpha-\text { Size } \\
\text { (Fig. 3A) }\end{array}$ & $\begin{array}{c}\alpha-\text { GRL } \\
\text { (Fig. 3C) }\end{array}$ & $\begin{array}{l}\text { Tpos-Size } \\
\text { (Fig. 3B) }\end{array}$ & $\begin{array}{l}\text { Tpos-GRL } \\
\text { (Fig. 3D) }\end{array}$ \\
\hline \multicolumn{5}{|l|}{ Population } \\
\hline Cranby & -0.09 & $-0.16(0.01)$ & -0.04 & $0.21(0.002)$ \\
\hline Dugout & 0.04 & 0.0 & 0.17 & 0.18 \\
\hline Ormond & $0.18(0.01)$ & -0.08 & $0.22(0.01)$ & 0.20 \\
\hline Paxton, benthic & $0.34(<0.001)$ & $0.28(0.001)$ & 0.14 & $-0.01(0.12)$ \\
\hline Paxton, limnetic & -0.11 & -0.06 & $0.41(<0.001)$ & 0.12 \\
\hline Priest, benthic & $0.2(<0.001)$ & $0.04(0.03)$ & 0.03 & $0.02(0.07)$ \\
\hline Priest, limnetic & -0.09 & -0.09 & $0.29(0.03)$ & 0.28 \\
\hline Kennedy & -0.16 & -0.18 & $0.32(0.006)$ & 0.27 \\
\hline \multicolumn{5}{|l|}{$F$ values } \\
\hline$F(\text { pop })_{1,387}$ & $113.9(<0.001)$ & $109.4(<0.001)$ & $14.2(<0.001)$ & $14.0(<0.001)$ \\
\hline$F$ (trait) 1.387 & $2.2(0.14)$ & $3.2(0.07)$ & $15.2(<0.001)$ & $24.3(<0.001)$ \\
\hline$F(\text { pop } \times \text { trait })_{7,387}$ & $4.9(<0.001)$ & $2.4(0.02)$ & $3.5(0.001)$ & $1.2(0.29)$ \\
\hline
\end{tabular}
following form (e.g., Tpos=GRL+Population+GRL $\times$ Population).

length supports the idea that rakers are an important functional trait that underlies individual specialization of sticklebacks (Svanback and Bolnick 2007), particularly in pelagic environments (Fig. 3D).

Unlike the benthics, sticklebacks from Cranby Lake (a population with an intermediate phenotype) exhibited strong patterns of individual specialization in both their habitat use and trophic position (Fig. 3C, D) but, similar to benthics, sticklebacks from Cranby Lake had no relationship between their body size and trophic position (Fig. 3B). This may result from a high degree of habitat specialization among individuals in Cranby Lake that masks population-level associations between stickleback body size and trophic position. Indeed, when we do not distinguish between benthics and limnetics, we see similarly weak relationships between stickleback morphometrics and isotopes in Paxton and Priest Lakes.

\section{DisCUSSION}

In accordance with our predictions (Fig. 1), we found that habitat specialization by sticklebacks is associated with a divergence in their trophic position. Stickleback populations specializing in pelagic environments had a higher trophic position (Figs. 2 and 3), and their trophic position increased more rapidly with their body size (Fig. 3B; Table 3). This can be explained by steeper relationships between body size and trophic position among pelagic prey, as compared to benthic prey (Diehl 1992; J. B. Shurin, B. Matthews, R. W. Markel, and S. Dick, unpublished manuscript). Furthermore, we found that body size and gill raker length are key traits that underlie individual specialization in the habitat use and trophic position of sticklebacks (Fig. 3, Table 3). In both allopatric and sympatric stickleback populations, individuals with longer gill rakers exploited more carbon from pelagic sources (Fig. 3C) and had a higher trophic position (Fig. 3D), particularly in pelagic habitats. We propose that incorporating a food web approach into studies of adaptive radiation, as we have done here using stable isotopes and morphometrics, may help identify the traits that underlie individual specialization and determine how the associations between morphology and other phenotypic traits, such as trophic position, might vary among habitats.

\section{Individual specialization of trophic position and habitat use by sticklebacks}

Comparative and experimental studies of adaptation have successfully linked phenotypic trait differences

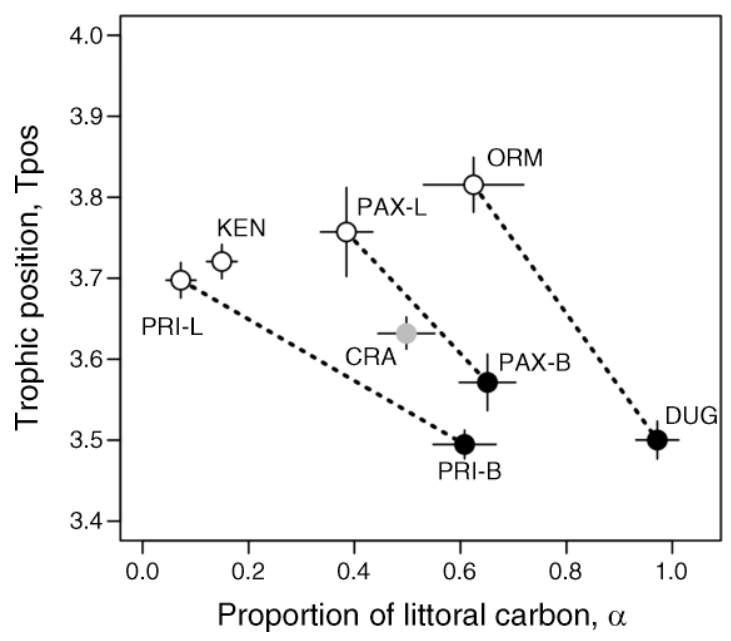

FIG. 2. Relationship between the average $( \pm$ SE) Tpos and $\alpha$ across lakes: Cranby Lake (CRA), Dugout Lake (DUG), Ormond Lake (ORM), Priest Lake (PRI), Paxton Lake (PAX), and Kennedy Lake (KEN). Solid black circles are the benthic phenotype, open circles are the limnetic phenotype, and the gray circle is the intermediate phenotype (as in Fig. 1). Limnetics (L) and benthics (B) for each sympatric population are indicated. 

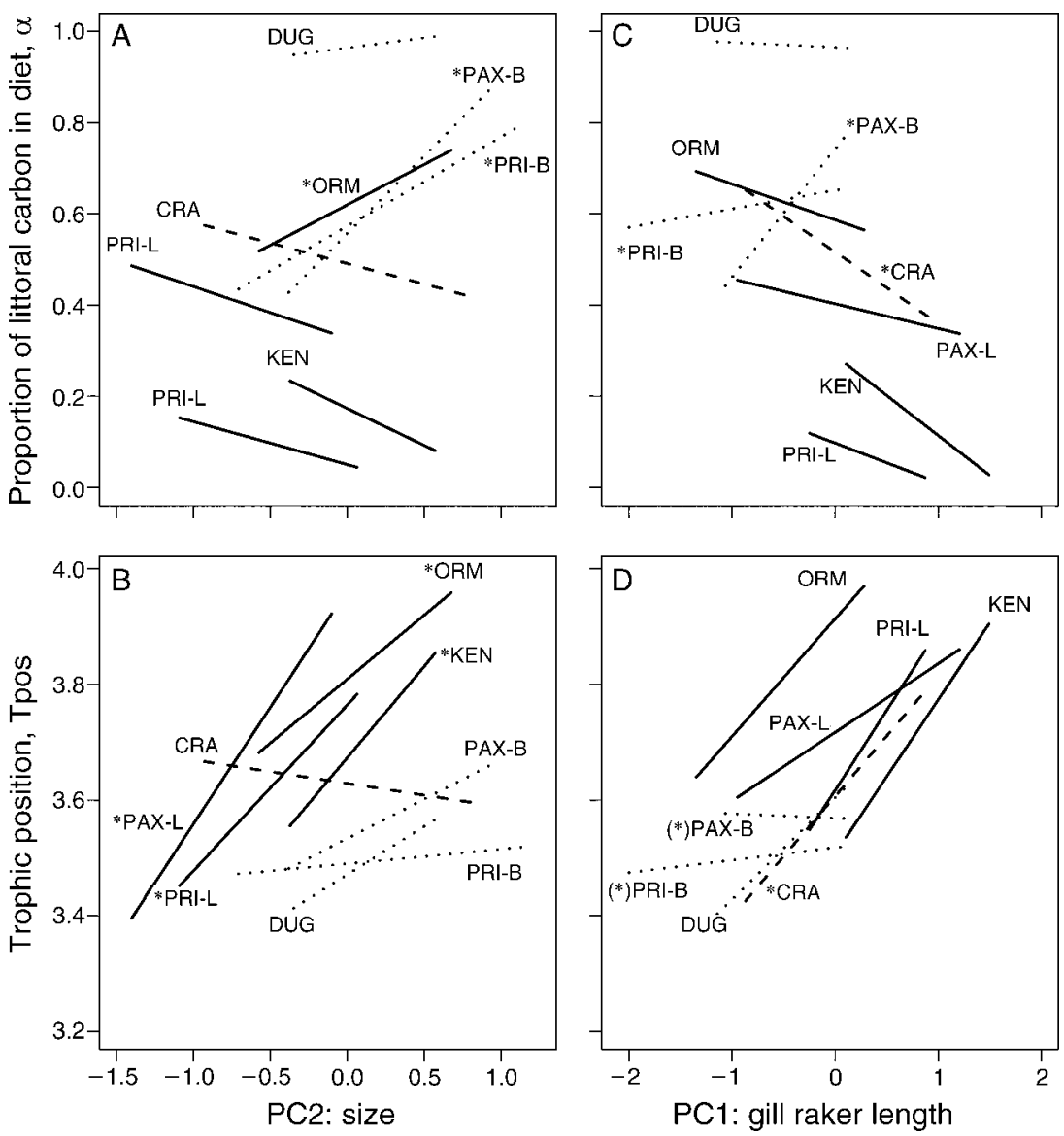

FIG. 3. These plots correspond to the predictions in Fig. 1. Solid lines are the "limnetic-like" phenotypes, dotted lines are the "benthic-like" phenotypes, and the dashed lines are the allopatric Cranby Lake population, as labeled in Fig. 2. The asterisks denote lines that are significantly different from a common slope $(P \leq 0.05$; Table 3$)$.

among individuals with particular ecological functions (Losos 1990, Wainwright 1994, Svanback and Eklov 2003). For example, in perch (Perca fluviatilis), deeper bodied individuals, which are characteristic of the littoral morph, grow faster on large littoral prey, while more streamlined pelagic individuals grow faster on the more conspicuous pelagic prey (Svanback and Eklov 2003). In sticklebacks, benthic species grow twice as fast in littoral compared to pelagic habitats (Schluter 1995), although the intraspecific relationships between morphology and growth rate are less clear. Assessing the correlation between morphology and isotopic variation provides an additional way to link foraging traits with long-term feeding performance in the field (Bolnick et al. 2003, Matthews and Mazumder 2004). Because isotopes record a longer dietary history than do gut contents, $\delta^{13} \mathrm{C}$ and $\delta^{15} \mathrm{~N}$ can be useful for estimating the degree of individual specialization in a population (Bolnick et al. 2003, 2008, Matthews and Mazumder 2004, Araujo et al. 2007). The strong relationship between gill raker length and trophic position in our study (Fig. 3D), both in allopatric populations and in limnetic species, suggests that gill raker length is a key trait underlying the individual specialization of sticklebacks to different trophic positions.

Divergence in gill raker morphology within and between populations is a common feature of the adaptive radiation of sticklebacks and other north and south temperate fishes (Schluter and McPhail 1993, Robinson and Wilson 1994, Lu and Bernatchez 1999, Ruzzante et al. 2003). Based on our observation that gill raker morphology underlies trophic position, it appears likely that trophic position itself is an emergent phenotypic trait that may be a target of selection. This emergent trait's expression is conditional on the community structure in which the individual occurs, and hence can exhibit substantial phenotypic plasticity across different environments. Variation in the trophic position of sticklebacks in natural lakes likely results from differences in the foraging abilities of sticklebacks in food webs with different size structure and species composition. Our study establishes a link between gill raker length and the trophic position of sticklebacks, but more work is needed to determine whether trophic position has a genetic basis, and whether it is under disruptive selection in the wild. In the current study, if 
we treat body size as a measure of relative fitness (following Bolnick 2004), and do an ANCOVA in the sympatric populations with stickleback species as a factor and $\alpha$ as a covariate, we find some evidence for divergent selection on habitat use (ANCOVA interaction term: Paxton, $F_{1,87}=18.4, P<0.001$; Priest, $F_{1,86}=$ $3.5, P=0.06)$. Using the same analysis we find no evidence for divergent selection with respect to trophic position (ANCOVA interaction term: Paxton, $F_{1,87}=$ $0.02, P=0.9$; Priest, $F_{1,87}=0.2, P=0.65$ ). While, there is limited power for these tests, and body size is not an ideal measure of fitness, it is important to consider how trophic position might evolve in sticklebacks. On one hand, it could evolve as a correlated response to evolution in gill raker length, which is both a heritable trait (Hermida et al. 2002) and often under disruptive selection in allopatric populations (Bolnick and Lau 2008). Alternatively, gill raker morphology could evolve in response to specialized foraging in pelagic environments on copepod prey that are difficult to catch but energetically rewarding (Yen and Strickler 1996). It is intriguing that the largest observed difference in trophic position was between sticklebacks from Ormond and Dugout, which show very little morphological divergence. This suggests that adaptation to different trophic positions may either be uncoupled from or precede morphological divergence in gill raker length, at least when there is strong gene flow between environments.

\section{Consequences of food web architecture for adaptive radiation}

Studies of adaptive radiations rarely consider how the architecture of food webs might influence niche divergence among species, or vice versa (Schluter 2000, Harmon 2009, Nosil and Harmon 2009). Lake food webs are structured such that consumers (particularly generalist predators) couple food chains that originate from primary production in pelagic and littoral habitats (Post 2002, Zanden and Vadeboncoeur 2002). The morphology and environmental conditions of lakes largely determine the relative size and productivity of each habitat type, which in turn may influence the strength of disruptive selection in stickleback populations (Bolnick and Lau 2008). The long-term stability of littoral and pelagic energy channels has likely enabled the evolution of pelagic and benthic forms in multiple species of postglacial fishes (Robinson and Wilson 1994, Landry et al. 2007). In addition to environmental controls on the form and persistence of selective environments in lakes, foraging adaptations by predators may further shape the underlying aquatic food web (Harmon et al. 2009). Our study lakes vary substantially in their depth (maximum depth: 1.8-145 m) and resemblance to ancestral oceanic (pelagic) foraging environments. Across this wide range of food webs and environmental conditions, niche divergence of sticklebacks has occurred both laterally, along a benthic and pelagic habitat axis, as well as vertically along a trophic position axis (Fig. 2). Further work is needed to determine whether such niche divergence is a by-product of environmental determinants of prey availability and composition, and whether the process of adaptation and speciation has feedback affects on the ecology and evolution of lake organisms (Harmon et al. 2009, Seehausen 2009).

The higher trophic position of sticklebacks in pelagic environments is consistent with the small size, fast turnover rate, and high trophic position of prey in pelagic habitats (Rooney et al. 2008). The prey of sticklebacks in pelagic food chains, such as copepods and Daphnia, are smaller and have higher rates of production per unit biomass (production:biomass ratios) than the typical prey in benthic food chains, such as amphipods and chironomids. As such, foraging on copepods might explain the higher trophic positions of sticklebacks in pelagic environments, and the positive relationship between their gill raker length and trophic position (Figs. 2 and 3). Copepods have a higher trophic position than other pelagic prey such as Daphnia or Diaphanosoma (Matthews and Mazumder 2005), and are a desirable prey item for sticklebacks. Previous gut content analyses and foraging experiments have shown that limnetics forage much more efficiently on copepods than do benthics (Schluter 1993), and in the breeding season copepods are a common prey item of limnetics (Schluter and McPhail 1992). Furthermore, in a recent mesocosm experiment, Paxton Lake limnetics reduced the abundance of $S$. oregonensis much more dramatically than either Paxton Lake benthics or Cranby Lake sticklebacks (Harmon et al. 2009). The omnivorous copepod $S$. oregonensis is common in our study lakes (Gahn et al. 1998, Bolnick et al. 2008) and, compared to other pelagic prey such as Daphnia, these copepods have a higher trophic position (Matthews and Mazumder 2005), are less vulnerable to predation (Yen and Strickler 1996), and have a higher nutritional value (Persson and Vrede 2006). Overall, these results suggest that copepods are a highly desired prey item for sticklebacks but are difficult to capture efficiently. We speculate that the adaptation of longer gill rakers may help sticklebacks forage more efficiently on copepods in pelagic environments. This hypothesis could be further tested by foraging experiments or field studies that couple gut content analysis with measurements of gill raker morphology.

It is interesting that benthics have a lower trophic position than limnetics, given that the benthic species is the more derived freshwater form of sticklebacks relative to ancestral oceanic populations. Marine, lake, and stream ecosystems have different predator-prey ratios (Brose 2006) and levels of omnivory (Thompson et al. 2007). As a result, upon colonization of freshwater habitats, sticklebacks have adapted to food webs with different size structures and trophic interactions. Though little is known about the diets of marine sticklebacks, the size structure of marine prey is more 
similar to the pelagic than the benthic prey communities of lakes (J. B. Shurin, B. Matthews, R. W. Markel, and $\mathrm{S}$. Dick, unpublished manuscript). A wider size structure of prey in freshwater environments, and/or a higher diversity of prey, may lead to lower trophic positions of sticklebacks in streams and littoral environments. Predators often exhibit weaker relationships between their body size and trophic position in foraging environments with a high diversity of prey and a weakly size structured food web (Layman et al. 2005). Comparing the trophic position and body shape of sticklebacks in freshwater and marine ecosystems may further reveal the traits that underlie evolution of stickleback specialization (Wund et al. 2008).

\section{ACKNOWLEDGMENTS}

The authors thank $T$. Ingram for his help with the morphology measurements and for helpful comments on the manuscript, as well as M. Deagle, N. Bock, and J. Samodien for their help in the field, and S. Mazumder for the isotope analysis. We also thank D. Schluter for his help with the project, and Paula Ramsay for her comments on the manuscript. This work was supported by a National Science Foundation grant DEB0412802 to D. I. Bolnick, a NSERC CGS and PDF to B. Matthews, and NSERC Disvovery and IRC grants to A. Mazumder.

\section{Literature Cited}

Abrams, P. A., C. Reuffler, and G. Kim. 2008. Determinants of the strength of disruptive and or divergent selection arising from resource competition. Evolution 62(7):1571-1586.

Araujo, M. S., D. I. Bolnick, G. Machado, A. A. Giaretta, and S. F. dos Reis. 2007. Using delta C-13 stable isotopes to quantify individual-level diet variation. Oecologia 152(4): 643-654.

Blumenshine, S. C., D. Lodge, and J. R. Hodgson. 2000. Gradient of fish predation alters body size distributions of lake benthos. Ecology 81:374-386.

Bolnick, D. I. 2004. Can intraspecific competition drive disruptive selection? An experimental test in natural populations of sticklebacks. Evolution 58(3):608-618.

Bolnick, D. I., E. J. Caldera, and B. Matthews. 2008. Evidence for asymmetric migration load in a pair of ecologically divergent stickleback populations. Biological Journal of the Linnean Society 94:273-287.

Bolnick, D. I., and O. L. Lau. 2008. Predictable patterns of disruptive selection in stickleback in postglacial lakes. American Naturalist 172:1-11.

Bolnick, D., R. Svanback, J. Fordyce, L. Yang, J. Davis, C. Hulsey, and M. Forister. 2003. The ecology of individuals: incidence and implications of individual specialization. American Naturalist 161:1-28.

Brose, U. e. a. 2006. Consumer-resource body-size relationships in natural food webs. Ecology 87:2411-2417.

Diehl, S. 1992. Fish predation and benthic community structure: the role of omnivory and habitat complexity. Ecology 73:1646-1661.

Duffy, J. E., B. J. Carinale, K. E. France, P. B. McIntyre, E. Thebault, and M. Loreau. 2007. The functional role of biodiversity in ecosystems: incorporating trophic complexity. Ecology Letters 10(6):522-538.

Dunne, J. A. 2005. The network structure of food webs. Pages 27-86 in M. Pascual and J. A. Dunne, editors. Ecological networks: linking structure to dynamics in food webs. Oxford University Press, Oxford, UK.

Fryer, G., and T. Iles. 1972. The cichlid fishes of the Great Lakes of Africa. Oliver and Boyd, Edinburgh, UK.
Gahn, D., K. D. Hyatt, and J. D. McPhail. 1998. Benefits and costs of vertical migration by the freshwater copepod Skistodiaptomus oregonensis: testing hypotheses through population comparison. Canadian Journal of Fisheries and Aquatic Sciences 55:1338-1349.

Grant, P. R. 1986. Ecology and evolution of Darwin's finches. Princeton University Press, Princeton, New Jersey, USA.

Harmon, L. J., B. Matthews, S. D. Roches, J. M. Chase, J. B. Shurin, and D. Schluter. 2009. Evolutionary diversification in stickleback affects ecosystem functioning. Nature 458:11671170.

Hatfield, T., and D. Schluter. 1999. Ecological speciation in sticklebacks: environment-dependent hybrid fitness. Evolution 53(3):866-873.

Hermida, M., C. Fernandez, R. Amaro, and E. S. Miguel. 2002. Heritability and "evolvability" of meristic characters in a natural population of Gasterosteus aculeatus. Canadian Journal of Zoology 80:532-541.

Jones, L., L. Becks, S. P. Ellner, N. G. Hairston, T. Yoshida, and G. F. Fussmann. 2009. Rapid contemporary evolution and clonal food web dynamics. Philosophical Transactions of the Royal Society B 364:1579-1591.

Kiljunen, M., J. Grey, T. Sinisalo, C. Harrod, H. Immonen, and R. I. Jones. 2006. A revised model for lipid-normalizing $\mathrm{d} 13 \mathrm{c}$ values from aquatic organisms, with implications for isotope mixing models. Journal of Applied Ecology 43:12131222.

Knudsen, R., P.-A. Amundsen, R. Primicerio, A. Klemetsen, and P. Sorensen. 2007. Contrasting niche-based variation in trophic morphology within Arctic charr populations. Evolutionary Ecology Research 9(6):1005-1021.

Landry, L., W. F. Vincent, and L. Bernatchez. 2007. Parallel evolution of lake whitefish dwarf ecotypes in association with limnological features of their adaptive landscape. Journal of Evolutionary Biology 20(3):971-984.

Lavin, P. A., and J. D. McPhail. 1985. The evolution of freshwater diversity in the threespine stickleback (Gasterosteus aculeatus): site-specific differentiation of trophic morphology. Canadian Journal of Zoology 63(11):2632-2638.

Layman, C. A., K. O. Winemiller, D. A. Arrington, and D. B. Jepsen. 2005. Body size and trophic position in a diverse tropical food web. Ecology 86:2530-2535.

Loeuille, N., and M. Loreau. 2005. Evolutionary emergence of size-structured food webs. Proceedings of the National Academy of Sciences (USA) 102(16):5761-5766.

Losos, J. B. 1990. The evolution of form and function: morphology and locomotor performance in West Indian Anolis lizards. Evolution 44(5):1189-1203.

Losos, J. B., T. R. Jackman, A. Larson, K. de Queiroz, and L. Rodriguez-Schettino. 1998. Contingency and determinism in replicated adaptive radiations of island lizards. Science 279: $2115-2118$

Lu, G., and L. Bernatchez. 1999. Correlated trophic specialization and genetic divergence in sympatric lake whitefish ecotypes (Coregonus clupeaformis): support for the ecological speciation hypothesis. Evolution 53(5):1491-1505.

Martinez, N. D. 2005. Network evolution: exploring the change and adaptation of complex ecological systems over deep time. Pages 287-302 in M. Pascual and J. A. Dunne, editors. Ecological networks: linking structure to dynamics in food webs. Oxford University Press, Oxford, New Jersey, USA.

Matthews, B., and A. Mazumder. 2004. A critical evaluation of intrapopulation variation of delta c-13 and isotopic evidence of individual specialization. Oecologia 140(2):361-371.

Matthews, B., and A. Mazumder. 2005. Consequences of large temporal variability of zooplankton delta $\mathrm{n}-15$ for modeling fish trophic position and variation. Limnology and Oceanography 50(5):1404-1414.

McCann, K., J. B. Rasmussen, and J. Umbanhowar. 2005. The dynamics of spatially coupled food webs. Ecology Letters 8: 513-523. 
Nosil, P., and L. J. Harmon. 2009. Niche dimensionality and ecological speciation. Pages 127-155 in R. Butlin, J. Bridle, and D. Schluter, editors. Ecology and speciation. Cambridge University Press, Cambridge, UK.

Persson, J., and T. Vrede. 2006. Polyunsaturated fatty acids in zooplankton: variation due to taxonomy and trophic position. Freshwater Biology 51(5):887-900.

Polis, G. A., and D. R. Strong. 1996. Food web complexity and community dynamics. American Naturalist 147(5):813-846.

Post, D. M. 2002. Using stable isotopes to estimate trophic position: models, methods, and assumptions. Ecology 83: 703-718.

R Development Core Team. 2008. R 2.7.1. R Project for Statistical Computing, Vienna, Austria. 〈www.r-project.com

Robinson, B. W. 2000. Trade offs in habitat-specific foraging efficiency and the nascent adaptive divergence of sticklebacks in lakes. Behaviour 137:865-888.

Robinson, B. W., and D. S. Wilson. 1994. Character release and displacement in fishes: a neglected literature. American Naturalist 144(4):596-627.

Rooney, N., K. McCann, G. Gellner, and J. C. Moore. 2006. Structural asymmetry and the stability of diverse food webs. Nature 442(7100):265-269.

Rooney, N., K. S. McCann, and J. C. Moore. 2008. A landscape theory for food web architecture. Ecology Letters 11(8):867-881.

Rundle, H. D., S. M. Vamosi, and D. Schluter. 2003. Experimental test of predation's effect on divergent selection during character displacement in sticklebacks. Proceedings of the National Academy of Sciences (USA) 100(25):1494314948.

Ruzzante, D. E., S. J. Walde, V. E. Cussac, P. J. Macchi, M. F. Alonso, and M. Battini. 2003. Resource polymorphism in a Patagonian fish Percichthys trucha (Percichthyidae): phenotypic evidence for interlake pattern variation. Biological Journal of the Linnean Society 78:497-515.

Schluter, D. 1993. Adaptive radiation in sticklebacks: size, shape, and habitat use efficiency. Ecology 74:699-709.

Schluter, D. 1995. Adaptive radiation in sticklebacks: trade-offs in feeding performance and growth. Ecology 76:82-90.
Schluter, D. 2000. The ecology of adaptive radiation. Oxford University Press, Oxford, UK.

Schluter, D., and J. D. McPhail. 1992. Ecological character displacement and speciation in sticklebacks. American Naturalist 140(1):85-108.

Schluter, D., and J. D. McPhail. 1993. Character displacement and replicate adaptive radiation. Trends in Ecology and Evolution 8(6):197-200.

Schluter, D., and L. M. Nagel. 1995. Parallel speciation by natural selection. American Naturalist 146(2):292-301.

Seehausen, O. 2009. Speciation affects ecosystems. Nature 458: $1122-1123$

Svanback, R., and D. I. Bolnick. 2007. Intraspecific competition drives increased resource use diversity within a natural population. Proceedings of the Royal Society B 274(1611): 839-844.

Svanback, R., and P. Eklov. 2003. Morphology dependent foraging efficiency in perch: a trade-off for ecological specialization? Oikos 102(2):273-284.

Thompson, R. M., M. Hemberg, B. M. Starzomski, and J. B. Shurin. 2007. Trophic levels and trophic tangles: the prevalence of omnivory in real food webs. Ecology 88:612617.

Vamosi, S. M., and D. Schluter. 2004. Character shifts in the defensive armor of sympatric sticklebacks. Evolution 58(2): 376-385.

Wainwright, P. C. 1994. Functional morphology as a tool in ecological research. Pages 42-59 in P. C. Wainwright and S. M. Reilly, editors. Ecological morphology. Chicago University Press, Chicago, Illinois, USA.

Wund, M. A., J. A. Baker, B. Clancy, J. L. Golub, and S. A. Foster. 2008. A test of the "flexible stem" model of evolution: ancestral plasticity, genetic accommodation, and morphological divergence in the threespine stickleback radiation. American Naturalist 172:449-462.

Yen, J., and J. R. Strickler. 1996. Advertisement and concealment in the plankton: what makes a copepod hydrodynamically conspicuous. Invertebrate Biology 3:191-205.

Zanden, M. J. V., and Y. Vadeboncoeur. 2002. Fishes as integrators of benthic and pelagic food webs. Ecology 83: 2152-2161.

\section{APPENDIX}

Location, size, and depth of stickleback lakes (Ecological Archives E091-072-A1). 